\title{
Evidence and clinical outcomes of adult soft tissue sarcomas of the extremities treated with adjuvant high-dose-rate brachytherapy - a literature review
}

\author{
Antonio Cássio Assis Pellizzon, MD, PhD \\ Department of Radiation Oncology, AC Camargo Cancer Center, São Paulo, Brazil
}

\begin{abstract}
The treatment strategies for adult soft tissue sarcomas of the extremities place an emphasis on local control, maintenance of limb function, and quality of life. Surgery is the mainstay of treatment for soft tissue sarcomas. Radiotherapy and chemotherapy are also both important treatments used in these patients to optimize the outcomes of limb sparing surgery. Compared to external beam radiation therapy, brachytherapy has the advantage of delivering a concentrated dose to the tumor, whilst sparing the normal tissues. Consequently, early and late complications such as bone fractures and subcutaneous fibrosis are potentially avoided by using brachytherapy. The evidence and clinical outcomes of HDR brachytherapy in soft tissue sarcomas of the extremities are described in this paper by means of a literature review.
\end{abstract}

J Contemp Brachytherapy 2014; 6, 3: 318-322

DOI: $10.5114 /$ jcb.2014.45758

Key words: brachytherapy, high-dose-rate, limb sparing surgery, radiotherapy, soft tissue sarcoma.

\section{Purpose}

Soft tissue sarcoma is a group of solid tumors with several anatomical and morphologic characteristics, arising in the mesenchymal support tissue. Evolving treatment strategies for adult patients with primary soft tissue sarcoma of the extremities (ESTS) emphasize local control (LC) with maintenance of limb function and quality of life (QOL). Although surgery remains the mainstay of therapy for primary ESTS, limited surgery alone has resulted in excessively high rates of local recurrence (LR). Both chemotherapy (CHT) and radiotherapy (RT) have been extensively used in the treatment of these tumors in attempts to optimize limb sparing surgery (LSS). Despite these advances, LR still occurs frequently especially in patients with marginal resections, positive surgical margins, large tumors at presentation, and re-resections of previously treated lesions [1]

In general for ESTS, the 1-year and 5-year estimated overall survival rates (OS) are around $80 \%$ and $60 \%$, respectively, when an aggressive surgical approach including amputation is used [2]. Studies investigating LSS combined with external beam radiation therapy (EBRT) showed similar rates of disease-free survival (DFS) and OS. The first prospective, randomized trial evaluating less aggressive surgical procedures was published in the 1980 's, and compared patients who had undergone amputation with those who had LSS combined with EBRT.
The study showed similar rates of DFS and OS in both patient groups [3].

In contrast to EBRT, which uses wide fields to generously encompass the surgical bed, brachytherapy (BT) concentrates the dose in the immediate vicinity of the surgical bed. Consequently, BT can potentially better spare normal tissues and avoid complications such as bone fractures, severe subcutaneous fibrosis or distal lymphedema [4]. Brachytherapy can be given by either low- (LDR) or high-dose-rate (HDR), and is an alternative to an EBRT boost. In some cases, BT can even substitute EBRT [5]. Furthermore, the use of BT does not preclude the addition of EBRT, which can complement the dose given by HDR when recommended.

For this literature review, a PubMed search was performed using the mesh terms "extremities soft tissue sarcoma brachytherapy". This search resulted in 100 articles. The following articles were excluded: case reports (13), technique descriptions (47), and duplicates (14). The remaining 26 articles are the subject of this review.

\section{Literature review}

The first studies evaluating LSS for ESTS began in the early 1980's. One prospective randomized trial investigated efficacy outcomes in patients treated with amputation compared to those treated with LSS combined with EBRT. Disease-free survival and OS rates were similar in 
both groups [3]. With different strategies yielding similar OS, recommendations for the use of RT may depend primarily on QOL issues and individual patient risks. Data from surgical series have shown that marginal excision alone of ESTS is associated with a local recurrence rate of $60 \%$ to $90 \%$. In contrast, a wide or compartmental radical excision, which often results in poor limb function, reduces the local recurrence rate to $10-30 \%[3,6,7]$.

A more recent, prospective randomized study published at the end of the 1990's, looked at the effect of adjuvant postoperative EBRT in patients with high and low grade extremity tumors. The results showed that there was a statistically significant reduction in LR in all patients regardless of tumor grade. Ninety-one patients with highgrade lesions were randomized; 47 patients received postoperative EBRT, and 44 did not. With a median follow-up of 9.6 years, a highly significant decrease $(p=0.0028)$ in the probability of LR was seen in the EBRT group, but no difference in OS was shown. Fifty patients with low-grade lesions were randomized; 26 received postoperative EBRT and 24 did not. For these patients there was also a lower probability of LR $(p=0.016)$ in the EBRT group, again, with no difference in OS. This study indicated that although postoperative EBRT is highly effective in preventing $\mathrm{LR}$, it is associated with late complications that can impact QOL. The patients in the EBRT group had significantly worse limb strength, oedema, and range of motion, though these impairments were often transient [8].

Based on these studies, EBRT doses over 60 Gy are generally given after LSS. However, this dose level can result in functional impairment of the limb and, in some cases, complications that may lead to amputation [9]. The use of BT, given either by LDR or HDR, can be an alternative to EBRT. Brachytherapy enables a relatively high dose of radiation to be given to a limited amount of tissue, resulting in higher rates of LC and a lower incidence of side effects. It should be emphasized that HDR also has some additional advantages over LDR in terms of radiation dose distribution. With HDR, the dose distribution can be optimized by changing the dwell positions and times of the source, with negligible radiation exposure to medical personnel [5]

The first prospective randomized study using BT instead of EBRT to treat the tumor bed after LSS was pub- lished by Pisters et al. [10]. This study verified that adjuvant LDR had an impact on LC in patients with high-grade tumors, but no effect in patients with low-grade tumors. This difference in results seen in patients with low-grade tumors raised the possibility that LDR could be clinically inferior to EBRT in terms of LC. The estimated LR rate of patients with high-grade tumors in the study who did not receive adjuvant LDR was $22 \%$ at 10 years. It is important to note that patients in whom complete tumor resection could not be accomplished without ablating all limb function were not included in this trial. Therefore, the conclusions of this study are relevant only for patients who undergo local excision with negative margins, and the results should not be extrapolated to patients with inadequate tumor resections.

When RT is indicated, it is known that reductions in normal tissue doses can decrease the probability of growth deformity. BT is therefore a good treatment option that can be used either alone or in combination with EBRT. Interstitial BT is a modern radiation treatment approach that has been improved over time by the incorporation of computed dosimetry and image guidance. HDR has also facilitated the broader use of interstitial BT, because it is a fast, reliable, and relatively inexpensive method.

The experience with HDR for ESTS is limited and is shown in Table 1. Some predictive factors for both LC and complications have already been reviewed in the literature and are discussed below.

\section{Resection margins}

Previous studies have suggested that recurrent tumor treatment and surgical margins have the greatest impact on local recurrence [12,13]. Martinez-Monge et al. [14] evaluated the treatment outcomes of 25 patients treated with surgical resection and subsequent HDR treatments up to 32 Gy. The HDR dose was prescribed based on the presumed amount of remaining residual disease. Surgical margins equal to or greater than $10 \mathrm{~mm}$ were classified as negative, and any negative margin less than $10 \mathrm{~mm}$ as close. The HDR dose was 4 Gy twice daily to a total dose as follows: 16 Gy total dose for negative margins, $24 \mathrm{~Gy}$ for close/microscopically positive margins, and $32 \mathrm{~Gy}$ for grossly involved margins. Radiation treatment was com-

Table 1. Recent published data

\begin{tabular}{llllccc} 
Author & Year & FU & $N$ & Treatment & LC (\%) & Complication > G2 (\%) \\
\hline Koizumi [15] & 1999 & 30 & 16 & HDR & 50.0 & 6.0 \\
\hline Pellizon [5] & 2000 & 35 & 27 & HDR + EBRT & 85.2 & 3.5 \\
\hline Chun [25] & 2001 & 31 & 17 & HDR + EBRT & 100 & NR \\
\hline Martinez-Monge [14] & 2005 & 23 & 25 & HDR + EBRT & 23.0 & 28.0 \\
\hline Pohar [24] & 2007 & 24 & 17 & HDR + EBRT & 94.0 & 18.0 \\
\hline Itami [18] & 2010 & 50 & 26 & HDR & 78.0 & 11.5 \\
\hline Petera [17] & 2010 & 43 & 45 & HDR & 55.0 & 4.4
\end{tabular}

FU - median follow-up time, $N$ - number of patients, HDR - high-dose-rate brachytherapy, EBRT-external beam radiation therapy, LC - local control, G2 - grade 2 RTOG complication 
pleted with EBRT (45 Gy in 25 daily fractions of 1.8 Gy) 4-5 weeks after the surgical procedure.

In the results, the authors noted that resection margins were negative in 6 patients $(24.0 \%)$, close/microscopically positive in $18(72.0 \%)$, and grossly involved in $1(4.0 \%)$. Three patients failed in the anatomical region treated, but relapse sites were not adjacent to the area treated with HDR. After a median follow up of 23.2 months (range 2.8-48.0), the 4-year local and regional control rates were $100 \%$ and $80.5 \%$, respectively. Four-year OS was $78.2 \%$.

Koizumi et al. [15] reported the results of 16 lesions treated with HDR BT between 1992 and 1996. The lesion resection margins were classified as follows: intracapsular (5 lesions), marginal (5 lesions), and wide (6 lesions). After a median follow up of 30 months, the LC was $75 \%$ at 1 year and $48 \%$ at 2 years. Ultimate LC was achieved in $8(50 \%)$ out of 16 lesions. Of the 8 uncontrolled lesions, $5(63 \%)$ had intracapsular (macroscopically positive) resection margins, whilst all the 8 controlled lesions (100\%) had marginal (microscopically positive) or wide (negative) margins. The total dose was 40-50 Gy given in 7 to 10 fractions twice daily over 4 to 7 days. With 3 recurrences occurring in the periphery or outside of the irradiated region, the authors concluded that a better LC may be achieved if the exact margin of residual tumor could be identified and boosted.

Petera et al. [16] reviewed the outcomes of 10 adult patients treated with HDR in combination with EBRT. Surgical margins were negative in 7 cases, close in 2 cases, and positive in 1 case. Doses of 3 Gy per fraction twice daily at $10 \mathrm{~mm}$ were prescribed, with total doses ranging from 18 to 30 Gy for HDR and 40-50 Gy for EBRT. With a median follow-up of 46 months they noted that LC was achieved in all 10 patients. Distant metastases occurred in 2 cases. One patient was disease free after salvage surgery and chemotherapy, and one patient died of lung disease progression 14 months after HDR. In one case, a subcutaneous fistula occurred after radiotherapy and was cured by surgical excision. Six patients experienced grade 1 or 2 fibrosis and in one case a mild peripheral neuropathy was recorded.

A more recent publication of this same group [17] involved 45 patients (17 primary and 28 recurrent lesions) treated between 1998 and 2007. Eleven patients were treated with HDR alone with a mean dose of 40 Gy (range: 30-54 Gy), and 34 were treated with a combination of HDR (mean dose: 24 Gy; 15-30 Gy), and EBRT (40-50 Gy). The LC rates were $100 \%$ for primary tumors and $64 \%$ in those patients treated for recurrent tumors $(p=0.004)$. The 5 -year actuarial OS and LC were $70 \%$ and $74 \%$, respectively.

Itami et al. [18] also published the results of 25 patients with 26 lesions treated with HDR as a single adjuvant modality. Thirteen lesions were marginal resections with positive surgical margins, and the remaining 50\% had very close margins. Fourteen lesions were treated for local recurrences after previous resections. The prescription dose was 36 Gy given in 12 fractions of 3 Gy twice daily. The 5-year LC observed was $78.2 \%$ and no LR was seen within the treated volume. Two groups were defined according to the margin status and previous op- erations. The first group included lesions with a positive margin and previous resections, whilst the second group included all remaining lesions. The 5-year LC was $43.8 \%$ and $93.3 \%$, respectively ( $p=0.004$ ), showing that dose escalation may impact results of LR for marginal or suboptimal resections.

\section{Time to initiate brachytherapy}

Brachytherapy has been noted to delay wound healing, significantly increasing the rate of patient morbidity. It has been implicated in prolonging the patient's hospital stay and increasing the cost and length of treatment. The application technique for interstitial BT has been modified over the past 40 years. These changes have improved the delivery of radiation to the tumor bed and decreased the associated morbidity rate. EBRT, which is usually administered 4 to 6 weeks after surgery, may miss the therapeutic interval that BT is able to capture. In addition, the 4 to 6 weeks of postoperative EBRT is circumvented by approximately 1 week (6 working days on a classical bid $36 \mathrm{~Gy} / 12$ fractions), thereby shortening the length of treatment [19]. The use of 3D planning with BT also enables better dose coverage of the tumor bed and skin sparing.

Ormsby et al. [20] reported that loading the interstitial catheters with a radioactive source on the first through to the fifth postoperative days resulted in a significant wound-complication rate of $48 \%$. As a result they changed their treatment policy, with catheters being loaded only five or more days after the operation. The wound complication rate was subsequently reviewed in 50 patients who underwent this new treatment strategy. Of the 21 patients receiving LDR, $14 \%$ had significant wound complications, a rate comparable to $10 \%$ of the 29 patients who did not receive radiation and had wound complications of similar severity. This demonstrated that the timing of radioactive source loading in the postoperative period is a major factor in radiation-induced wound-healing delay. This institution's experience suggests that 5 to 7 days post surgery is a safe time to load the interstitial catheters.

The association of wound complications with patient age, co-morbidities or treatment factors was studied by Aronowitz et al. [21] in a small series of 12 patients treated with HDR plus EBRT. The study results showed that there may be no relationship between wound healing and patient age, diabetes, width of excised skin, cross-sectional area of implant, sequencing of therapy or surgery-to-HDR interval.

\section{Tumor location}

It is postulated that tumors in the extremities do not correlate with a higher probability of complications. A publication by Delannes et al. [22] focusing on LDR treatments showed a correlation between the location of the tumor in the lower limb and early side effects $(p=0.003)$. The proximity of the tumor to neurovascular structures was correlated with late side effects $(p=0.009)$. In contrast to this, Kubo et al. [23] published the results of 7 patients treated between 1995 and 2000 for ESTS involving the neurovascular bundle. They were treated 
with LSS followed by fractionated HDR. Catheters were placed within the tumor bed directly upon the preserved neurovascular structures. Six patients received a total dose of $50 \mathrm{~Gy}$ with HDR whilst one patient received 30 Gy of HDR combined with 20 Gy of adjuvant EBRT. The 5-year actuarial OS, LC and disease specific survival rates were $83.3 \%, 83.3 \%$, and $68.6 \%$, respectively. None of the 7 patients developed HDR induced peripheral neuropathy or neurotoxicity.

\section{High-dose-rate compared to low-dose-rate}

Pohar et al. [24] compared the results of LDR and HDR. A group of 37 patients treated with LDR (20) or HDR (17) plus EBRT were evaluated and no significant difference in the 2-year LC (90\% with LDR and $94 \%$ for HDR) was found. The rate of grade 2-4 complications was $40 \%$ in the LDR group and $18 \%$ in the HDR group. The difference however was not statistically significant $(p=0.14)$, probably due to the relatively small number of patients in the cohort. Interestingly, on univariate analysis, suboptimal geometry of the implant was predictive of an increased complication rate in the LDR group. These results therefore confirm that optimization of dose distribution by varying the dwell time at each dwell position, as made possible by HDR, may compensate for suboptimal geometry and thus reduce the rate of complications.

\section{High-dose-rate and external beam radiation therapy}

Pellizzon et al. [7] have also published the results of 27 patients who presented with ESTS and were treated with LSS and HDR from 1993 to 1999. The postoperative HDR dose prescription ranged from $18 \mathrm{~Gy}$ to $36 \mathrm{~Gy}$, given in 3 to 6 Gy fractions, twice daily. External beam radiation therapy was used pre or postoperatively with a median dose of $40 \mathrm{~Gy}$ (30-55 Gy). The 5-year actuarial LC and OS observed were $85.2 \%$ and $93.7 \%$, respectively.

Chun et al. [25] examined the results of 17 patients with ESTS (11 primary and 6 recurrent) treated between 1995 and 1999 with HDR plus EBRT. On $6^{\text {th }}$ postoperative day, patients received HDR, 2-3 Gy per fraction twice daily up to 12-18 Gy, followed 3 weeks later by EBRT (36-60 Gy). With a median follow-up of 31 months, there was no local failure within the radiation field in any of the patients. One patient did require wound revision for delayed healing after HDR, but there was no neuropathy or significant fibrosis in any of the patients.

The American Brachytherapy Society [26] published a consensus statement for ESTS brachytherapy last year. They reported that factors which influence the selection of radiation treatment modalities to be employed in the adjuvant setting include tumor grade and size, prior surgeries, and tumor recurrence. They conclude that LDR, HDR, and pulsed-dose-rate radiation are all acceptable modalities to use for ESTS either alone or in combination with EBRT.

\section{Conclusions}

Results for HDR interstitial BT for the treatment of ESTS in adults are quite encouraging. The clinical equiv- alence between HDR schedules and LDR has gained more acceptance. High-dose-rate can definitively irradiate ESTS after surgery, either alone or in combination with EBRT. High-dose-rate also permits re-irradiation by strictly localizing the high dose radiation exposure. In addition, HDR provides the advantage of a rapid completion of local treatment, allowing for earlier initiation of systemic therapy when necessary. Prospective studies on the use of HDR in the adjuvant setting to treat ESTS are still necessary to define the optimal dose/fraction schedule.

\section{Disclosure}

Author reports no conflict of interest.

\section{References}

1. Zagars GK, Ballo MT, Pisters PW et al. Preoperative vs postoperative radiation therapy for soft tissue sarcoma: a retrospective comparative evaluation of disease outcome. Int J Radiat Oncol Biol Phys 2003; 56: 482-488.

2. Storm HH. Survival of adult patients with cancer of soft tissues or bone in Europe. EUROCARE Working Group. Eur J Cancer 1998; 34: 2212-2217.

3. Rosenberg SA, Tepper J, Glatstein E et al. The treatment of soft-tissue sarcomas of the extremities: prospective randomized evaluations of (1) limb-sparing surgery plus radiation therapy compared with amputation and (2) the role of adjuvant chemotherapy. Ann Surg 1982; 196: 305-315.

4. Viani GA, Novaes PE, Jacinto AA et al. High-dose-rate brachytherapy for soft tissue sarcoma in children: a single institution experience. Radiat Oncol 2008; 3: 9.

5. Pellizzon ACA, Nadalin W, Salvajoli JV et al. High dose rate brachytherapy as a boost in management of adult soft tissue sarcoma: results and complications. J. B. Int 2000; 17: 43-50.

6. DeLaney TF. Optimizing radiation therapy and post-treatment function in the management of extremity soft tissue sarcoma. Curr Treat Options Oncol 2004; 5: 463-476.

7. Trovik CS, Scanadinavian Sarcoma Group Project. Local recurrence of soft tissue sarcoma. A Scandinavian Sarcoma Group Project. Acta Orthop Scand 2001; 72: 1-31.

8. Yang JC, Chang AE, Baker AR et al. Randomized prospective study of the benefit of adjuvant radiation therapy in the treatment of soft tissue sarcomas of the extremity. I Clin Oncol 1998; 16: 197-203.

9. Stinson SF, DeLaney TF, Greenberg J et al. Acute and longterm effects on limb function of combined modality limb sparing therapy for extremity soft tissue sarcoma. Int J Radiat Oncol Biol Phys 1991; 21: 1493-1499.

10. Pisters PW, Harrison LB, Leung DH et al. Long-term results of a prospective randomized trial of adjuvant brachytherapy in soft tissue sarcoma. J Clin Oncol 1996; 14: 859-868.

11. Alekhteyar KM, Leung DH, Brennan MF et al. The effect of combined external beam radiotherapy and brachytherapy on local control and wound complications in patients with high-grade soft tissue sarcomas of the extremity with positive microscopic margin. Int J Radiat Oncol Biol Phys 1996; 36: 321-324.

12. Markhede G, Angervall L, Stener B et al. A multivariate analysis of the prognosis after surgical treatment of malignant soft-tissue tumors. Cancer 1982; 49: 1721-1733.

13. Collin CF, Friedrich C, Godbold J et al. Prognostic factors for local recurrence and survival in patients with localized extremity soft-tissue sarcoma. Semin Surg Oncol 1988; 4: 30-37. 
14. Martinez-Monge R, San Julian M, Amillo S et al. Perioperative high-dose-rate brachytherapy in soft tissue sarcomas of the extremity and superficial trunk in adults: initial results of a pilot study. Brachytherapy 2005; 4: 264-270.

15. Koizumi M, Inoue T, Yamazaki $\mathrm{H}$, et al. Perioperative fractionated high-dose rate brachytherapy for malignant bone and soft tissue tumors. Int J Radiat Oncol Biol Phys 1999; 43: 989-993.

16. Petera J, Neumanova R, Odrazka K et al. Perioperative hyperfractionated high-dose rate brachytherapy combined with external beam radiotherapy in the treatment of soft tissue sarcomas. Tumor 2005; 91: 331-334.

17. Petera J, Soumarová R, Růzicková J et al. Perioperative hyperfractionated high-dose rate brachytherapy for the treatment of soft tissue sarcomas: multicentric experience. Ann Surg Oncol 2010; 17: 206-210.

18. Itami J, Sumi M, Beppu Y et al. High dose rate brachytherapy alone in postoperative soft tissue sarcomas with close or positive margins. Brachytherapy 2010; 9: 349-353.

19. Devereux DF, Kent H, Brennan MF et al. Time dependent effects of adriamycin and x-ray therapy on wound healing in the rat. Cancer 1980; 45: 2805-2810.

20. Ormsby MV, Hilaris BS, Nori D et al. Wound complications of adjuvant radiation therapy in patients with soft tissue sarcomas. Ann Surg 1989; 210: 93-99.

21. Aronowitz JN, Pohar SS, Liu L et al. Adjuvant high dose rate brachytherapy in the management of soft tissue sarcoma: a dose-toxicity analysis. Am J Clin Oncol 2006; 29: 508-513.

22. Delannes M, Thomas L, Martel P et al. Low-dose-rate intraoperative brachytherapy combined with external beam irradiation in the conservative treatment of soft tissue sarcoma. Int J Radiat Oncol Biol Phys 2000; 47: 165-169.

23. Kubo T, Sugita T, Shimose $S$ et al. Nerve tolerance to highdose-rate brachytherapy in patients with soft tissue sarcoma: a retrospective study. BMC Cancer 2005; 5: 79.

24. Pohar S, Haq R, Liu L et al. Adjuvant high-dose-rate and lowdose-rate brachytherapy with external beam radiation in soft tissue sarcoma: a comparison of outcomes. Brachytherapy 2007; 6: 53-57.

25. Chun M, Kang S, Kim BS et al. High dose rate interstitial brachytherapy in soft tissue sarcoma: technical aspects and results. Jpn J Clin Oncol 2001; 31: 279-283.

26. Holloway CL, Delaney TF, Alektiar KM et al. American Brachytherapy Society (ABS) consensus statement for sarcoma brachytherapy. Brachytherapy 2013; 12: 179-190. 